\title{
Field Emission Studies on Methane- and Ethane-Adsorbed Carbon Nanotubes*
}

\author{
Hitoshi Nakahara, ${ }^{\dagger}$ Tetsuya Yamashita, and Yahachi Saito \\ Department of Quantum Engineering, Graduate School of Engineering, Nagoya University, Nagoya 464-8603, Japan
}

(Dated: Received 8 January 2010; Accepted 26 January 2010; Published 27 February 2010)

\begin{abstract}
Carbon nanotubes (CNTs) are known as one of the ideal electron emitter material. However, there are many challenges to solve for actual use such as emission current enhancement, emission current stability, life time extension, etc. In this work, methane and ethane gases are used to improve emission characteristics from CNT emitters. A CNT emitter used in this study is a bundle of multi-walled CNTs. In situ field emission microscopy is used to investigate gas effects on emission characteristics. From comparative experiments under different exposure conditions, it is found that gas exposure during field emission is effective to increase emission currents and the effects are comparable between two gases. It is also found that the effect caused by ethane gas has longer life time than that by methane gas. [DOI: 10.1380/ejssnt.2010.77]
\end{abstract}

Keywords: Field emission microscopy; Field emission measurements; Carbon nanotube; Methane; Ethane

\section{INTRODUCTION}

Carbon nanotubes (CNTs) have many kinds of unique electric, physical and chemical properties such as ballistic electric conduction, high stiffness, small tip radius, chemical stability, etc., so that there are many possible applications of CNTs. Because of its shape and high sublimation temperature, CNTs are known as one of the ideal electron emitter material. However, there are many challenges to solve for actual use such as emission current enhancement, emission current stability, life time extension, etc. Final goals of this study are applications as a large area electron source such as a field emission display, a field emission vacuum gauge, an electron shower and so on, all of them require higher emission current at lower applied voltage.

Electron emission from a CNT is strongly dependent on its tip surface structure and adsorbates [1-6]. Theoretical calculations [4] predict that methane gas adsorption onto a CNT tip increases work function of the surface, which results in decrease of emission current. However, experimental works of various hydro-carbon gas adsorption $[5,6]$ report increase of emission currents. In this paper, methane and ethane gases were used to improve emission characteristics from CNT emitters to clarify mechanisms of emission increase and to investigate optimum treatment conditions.

\section{EXPERIMENTAL}

Figure 1(a) shows a schematic illustration of a field emission microscopy (FEM) apparatus used in this experiment. The apparatus has a specimen exchange chamber (left-hand side of the figure) and a FEM chamber (righthand side of the figure) separated by a gate valve. The FEM chamber has a gas inlet variable leak valve to introduce methane or ethane gas. A CNT emitter mounted on a sample holder was placed about $30 \mathrm{~mm}$ away from a flu-

*This paper was presented at 7th International Symposium on Atomic Level Characterizations for New Materials and Devices, The Westin Maui Resort \& Spa, Hawaii, U.S.A., 6-11 December, 2009.

$\dagger$ Corresponding author: nakahara@nagoya-u.jp

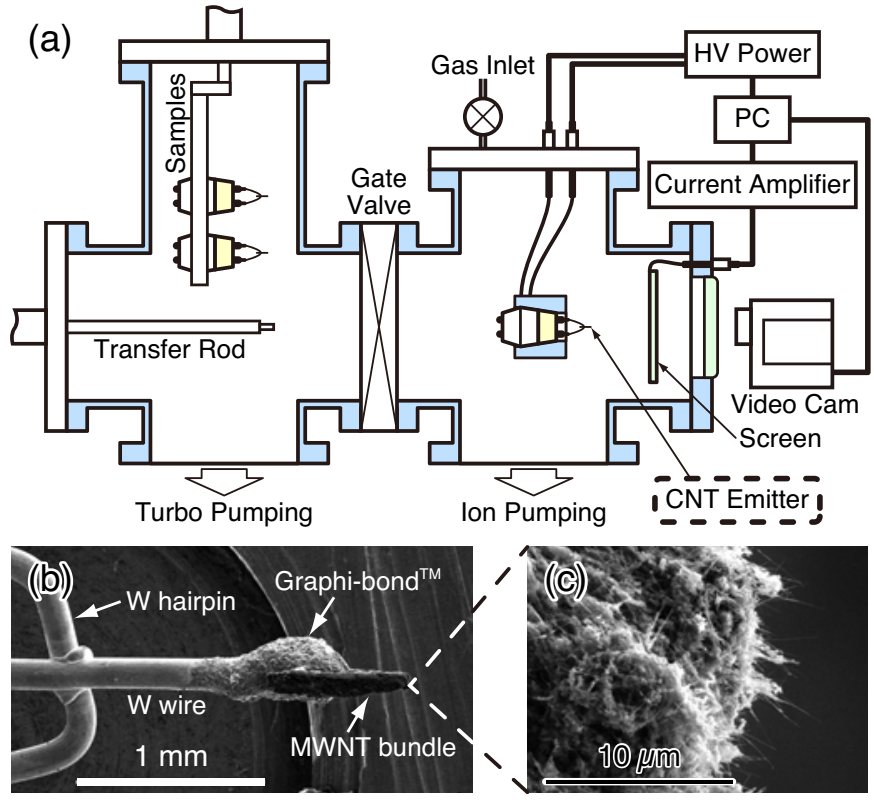

FIG. 1: (a) A schematic illustration of a FEM apparatus. (b) A SEM image of a CNT emitter. (c) An expanded SEM image of the CNT emitter tip.

orescent screen, and high voltage (HV; at most $\pm 700 \mathrm{~V}$ ) was applied to the CNT emitter. FEM patterns projected onto the screen was monitored by a video camera. A HV power supply was controlled by a personal computer (PC) and emission currents were monitored by a current amplifier. Both of FEM patterns and emission currents were recorded by the PC. Base pressure of the FEM chamber was $8 \times 10^{-8} \mathrm{~Pa}$.

CNTs used in this study were multi-walled CNTs (MWNTs) produced by catalyst free arc-discharge method under $4 \times 10^{4} \mathrm{~Pa}$ of He pressure. Diameters of the MWNTs were about $10 \sim 20 \mathrm{~nm}$, which was measured by a transmission electron microscopy. A bundle of MWNT with $\sim 0.1 \mathrm{~mm}$ diameter and $\sim 1 \mathrm{~mm}$ length was attached to a tungsten (W) wire by Graphi-bond (see a scanning electron microscopy (SEM) image of Fig. 1(b)). The W wire can be heated by direct current through a $\mathrm{W}$ hairpin on which the $\mathrm{W}$ wire was welded. As shown in an expanded SEM image (Fig. 1(c)), the tip of the bundle consists of many MWNT emitters sticking out from the bundle. Before starting experiments, a CNT emitter were 


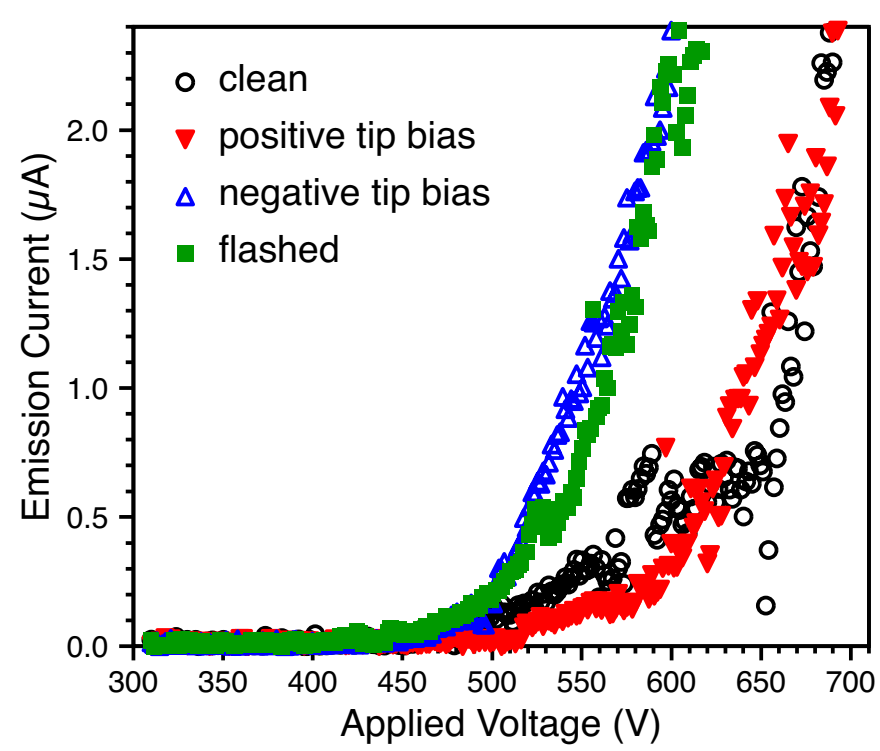

FIG. 2: $I-V$ curves for a clean emitter (open circles), after exposed to methane under positive tip bias (filled down triangles) and negative tip bias (open up triangles), and after a flashing process (filled squares). This is a series of experiments using an identical emitter.

annealed several times at about $700 \mathrm{~K}$ for few minutes to obtain a clean FEM pattern from MWNTs (pentagons pattern, see Ref. [1]).

Two kinds of exposure conditions, one is a positive tip bias condition and another is a negative one, were used upon metane/ethane gas exposure. Under the positive bias condition, $+700 \mathrm{~V}$ was applied to a CNT emitter against the screen. Apparently no field emission occurs under this condition. On the other hand, $-700 \mathrm{~V}$ was applied under the negative bias condition, which means field emission occurs during gas exposure. Vacuum pressure during methane/ethane gas exposure was $10^{-6}$ to $10^{-5} \mathrm{~Pa}$ which was controlled by the gate valve and the variable leak valve. To measure $I-V$ curves of gas exposed emitter, introduced gases were evacuated.

\section{RESULTS AND DISCUSSIONS}

Figure 2 shows $I$ - $V$ curves before methane exposure (open circles), under positive bias (filled down triangles), under negative bias exposure to methane (open up triangles), and after flashing at about $1200 \mathrm{~K}$ of the methane exposed emitter (filled squares). This is a series of experiments using an identical emitter. As shown in the figure, positive biased exposure have little or no effect on $I-V$ characteristic, while under the negative bias condition, the $I-V$ curve shifts toward lower voltage by $\sim 90 \mathrm{~V}$. The $I-V$ curve does not change by flash heating of the emitter. Fig. 3 shows corresponding FEM patterns for $I$ $V$ curves in Fig. 2. As shown in a white broken circle in each patten, FEM before exposure (a) and that after positively biased exposure (b) show clear pentagons pattern which means clean CNT cap remains [1]). After negatively biased exposure (c), the pattern changed to disordered one which shows adsorption of methane molecules on the CNT tip. The disordered pattern remains after the
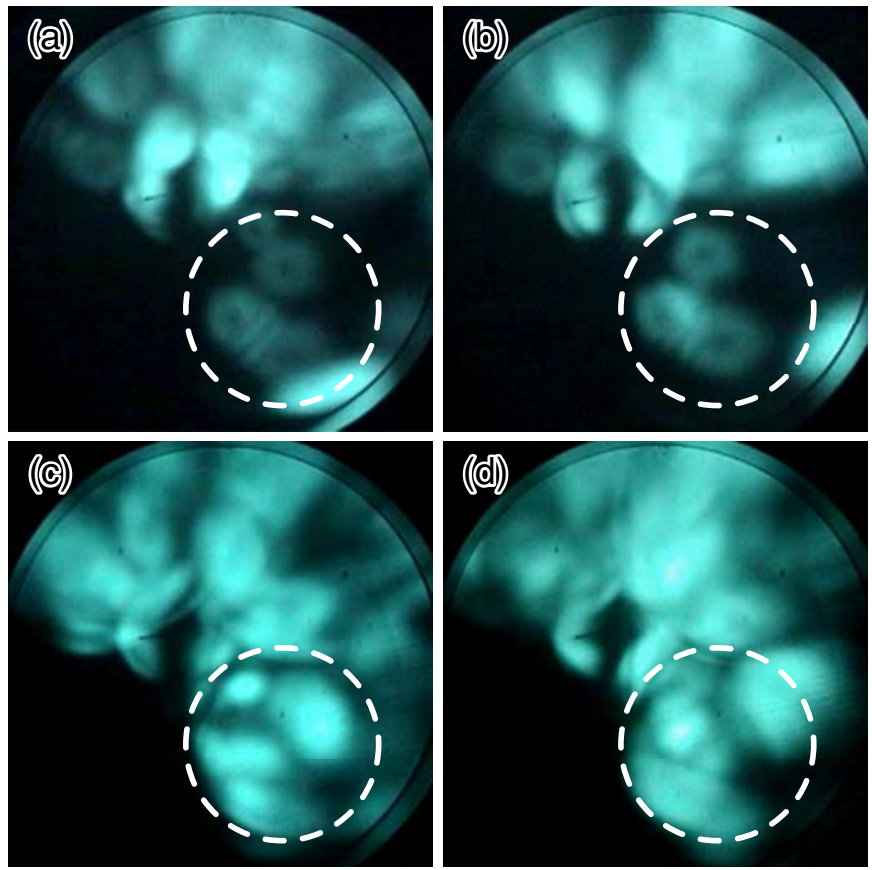

FIG. 3: FEM patterns of (a) a clean emitter, (b) after exposed to methane under positive tip bias, (c) after exposed to methane under negative tip bias, and (d) after a flashing process. This is a series of experiments using an identical emitter.

flashing process (d), or the tip could not be cleaned by the flashing. Both of the $I-V$ curves and FEM patterns indicate that positively biased exposure does not affect the CNT tip structure, and that relatively strong reaction between CNT cap and methane molecules occurred by the negative biased exposure.

Figure 4 is similar result as Fig. 2 except that the gas used was ethane. Unlike the methane case, $I-V$ curve shifted toward lower voltage by $\sim 35 \mathrm{~V}$ after positively bi-

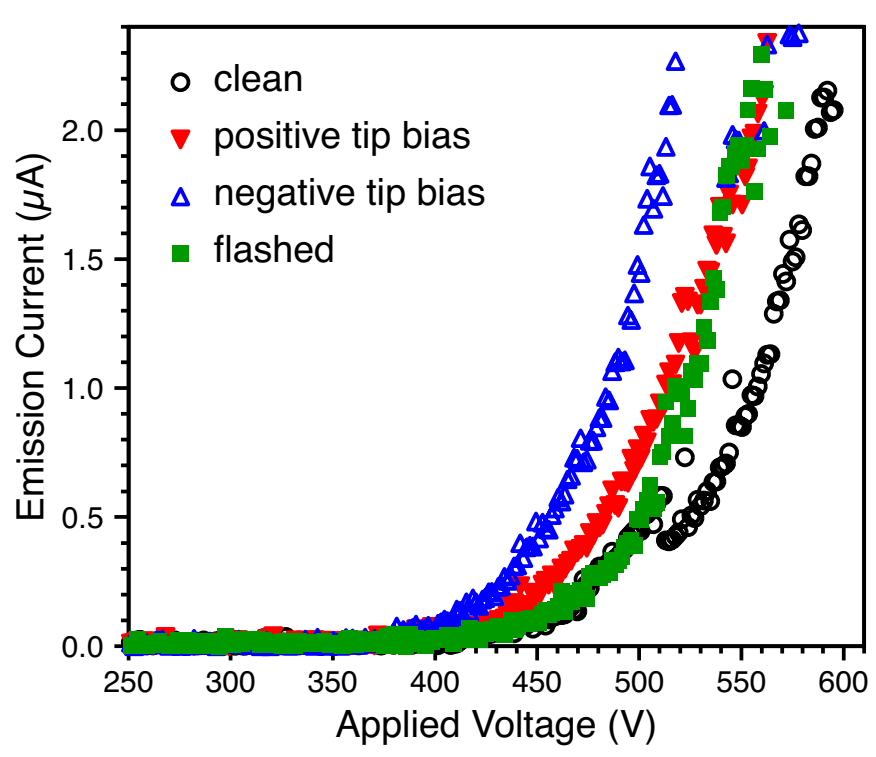

FIG. 4: $I-V$ curves for a clean emitter (open circles), after exposed to ethane under positive tip bias (filled down triangles) and negative tip bias (open up triangles), and after a flashing process (filled squares). This is a series of experiments using an identical emitter. 
ased exposure. The curve shifted toward lower voltage again by $\sim 35 \mathrm{~V}$ after negatively biased exposure. After flashing process, the $I-V$ curve move back toward higher voltage by $\sim 35 \mathrm{~V}$. It is observed from repetitive experiments, that the tendency explained above is the same but the shift voltage varies about $\pm 15 \mathrm{~V}$.

Both of methane and ethane gases have similar effects, i.e. gas exposure causes increase in emission currents or lower threshold voltages by several tens volts. Theoretical calculation [4] predicts suppression of emission current, however, it only takes work functions into account, but not field enhancement factors nor emission areas. As shown in Fig. 3(c), bright spots increased in the FEM pattern, which indicates increase of emission sites. In case of a clean MWNT, it is well known that only nearby areas of five-memberd-rings (5MR) can be act as emission sites $[1,7]$. It is considered that gas molecules can adsorb both on $5 \mathrm{MR}$ and $6 \mathrm{MR}$ (relative area of $6 \mathrm{MR}$ is much larger than that of $5 \mathrm{MR}$ ), which causes increase in field emission current despite of a larger work function.

For the bias polarity during exposure, it is considered that emitted electrons ionize gas molecules and the negative CNT tip attracts positively charged ions. Then the ions attack the CNT tip with $700 \mathrm{eV}$ of kinetic energy, which is expected to cause chemical bonding between the CNT and the molecule. In the case of positive bias, most of molecules which come onto the CNT tip are neutral with thermal energy of room temperature $(\sim 26 \mathrm{meV})$. Therefore, in this case, weak physisorption takes place and the physisorped molecule easily desorb from the tip surface during field emission. It is consistent with the result in Fig. 3(b) that a clean FEM pattern still observed after positive biased exposure. After flashing of negative bias exposed samples (both for methane and ethane cases) at about $1200 \mathrm{~K}$, both of $I-V$ curves and FEM patters did not recover as its clean state. It is reported that adsorption energies between graphite and methane/ethane molecules are $\sim 150 \mathrm{meV}$ and $\sim 202 \mathrm{meV}$ respectively [8]. Comparing with the flashing temperature of $1200 \mathrm{~K}$ (its thermal energy is about $103 \mathrm{meV}$ ), it is obvious that the flashing temperature is insufficient to remove chemically adsorbed molecules from graphite surface. It is also a evidence that chemisorption takes place when gases are exposed under negative bias condition.

Figure 5 and Fig. 6 show results of life time tests. Open circles show $I-V$ curves before gas exposure and open triangles show the curves after negatively biased exposure. In place of flashing process, samples are left under FE conditions (emission current 1 2 $\mu \mathrm{A}$ ) for 3 hours (filled diamonds). During the emission, methane/ethane gases had been evacuated. For methane case (Fig. 5), $I$ - $V$ curve after $3 \mathrm{~h}$ FE recovered to its clean state. However in the case of ethane (Fig. 6), the curve recovers little and the gas exposure effect remains. It is reported [9] that CNT tip temperature during field emission could reach to $2000 \mathrm{~K}$ which corresponds to a thermal energy of $172 \mathrm{meV}$. Although tip temperature strongly depends on CNT radius and length, this thermal energy is comparable to adsorption energies of methane/ethane molecules. Thus it is considered that methane molecules, which has lower adsorption energy, desorbed from the CNT tip quicker than ethane molecules. In general, heavier molecule has larger adsorption energy [8], heavier molecule is expected to have

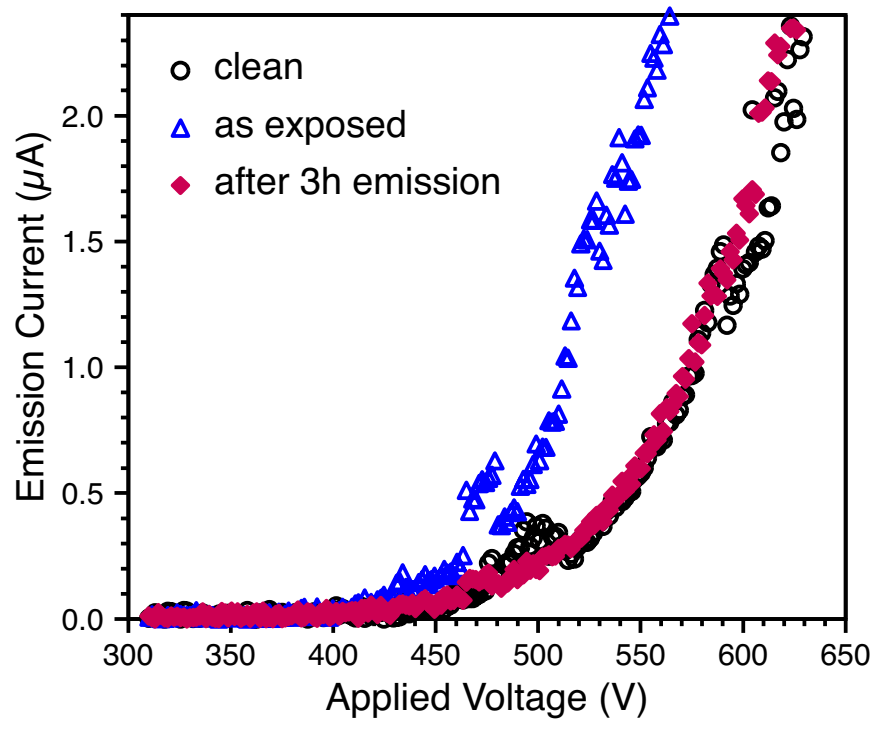

FIG. 5: $I-V$ curves for a clean emitter (open circles), as exposed to methane (open triangles), and after 3 hours of field emission (filled diamonds). This is a series of experiments using an identical emitter.

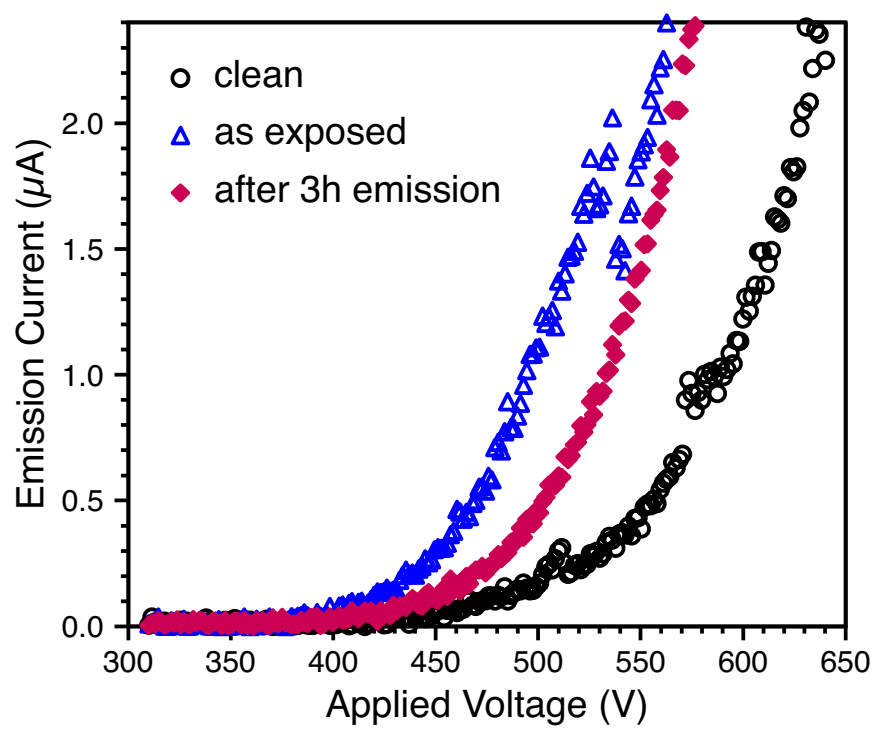

FIG. 6: $I-V$ curves for a clean emitter (open circles), as exposed to ethane (open triangles), and after 3 hours of field emission (filled diamonds). This is a series of experiments using an identical emitter.

longer life time for field emission enhancement.

It was shown that both methane and ethane can be used as field emission enhancer, however, emission stability became worse than a clean one. In our experiment, a large bundle of MWNTs was used and it is possible to act as a storage of gas. Therefore gas molecules from the storage causes instability after evacuating the gas. However, for the purpose of large area electron source as a field emission display, instability of individual emitter is statistically-balanced by $\sqrt{N}$ law (here $N$ is a number of individual emitters). Assuming that one pixel of a electron source consists of 10,000 CNTs, instability decreases by $1 / 100$. Thus we are optimistic about emission stability of single CNT emitter. 


\section{SUMMARY}

Methane and ethane molecules adsorption effects on field emission characteristics of CNTs are investigated and the following results were obtained. Both of methane and ethane molecules make chemical bonding under negatively biased exposure condition, but only physisorption occurs under positively biased condition. It is considered that gas molecules are ionized by field emitted electrons under negative bias condition and the positively charged molecular ions attack a CNT tip to make chemical bonding.

Molecular adsorption on a CNT tip causes enhancement in field emission currents. The enhancement effects by methane and ethane gas are comparable and no significant difference was observed.

Flashing of a tip at about $1200 \mathrm{~K}$ can not remove ad- sorbed methane/ethane molecules, while 3 hours of FE can removed methane molecules completely from a CNT tip. Ethane molecules remains after 3 hours of FE, which is considered that ethane has strong binding energy than methane.

\section{Acknowledgments}

Authors wish to thank to Dr. Huarong Liu of Nagoya University for his helpful discussion about theoretical analyses of $I-V$ characteristics. We also acknowledge a financial support from the Ministry of Education, Culture, Sports, Science and Technology Japan (Grants-in-Aids for Scientific Research on Priority Areas, 19054007).
[1] Y. Saito, K. Hata, and T. Murata, Jpn. J. Appl. Phys. 39, L271 (2000).

[2] A. Wadhawan, R. E. Stallcup II, K. F. Stephens II, J. M. Perez, and I. A. Akwani, Appl. Phys. Lett. 79, 1867 (2001).

[3] K. S. Yeong and J. T. L. Thong, Appl. Surf. Sci. 233, 20 (2004).

[4] Z. Li and C.-Y. Wang, Chem. Phys. 330, 417 (2006).

[5] L. M. Sheng, P. Liu, Y. M. Liu, L. Qian, Y. S. Huang, L. Liu, and S. S. Fan, J. Vac. Sci. Technol. A 21, 1202 (2003).

[6] A. M. Fennimore, D. H. Roach, G. A. Wilson, F. M. Pel- licone, and L. T. Cheng, Appl. Phys. Lett. 92, 213108 (2008).

[7] H. Nakahara, Y. Kusano, T. Kono, and Y. Saito, Appl. Surf. Sci. 256, 1214 (2009).

[8] T. R. Rybolt, K. A. Ziegler, H. E. Thomas, J. L. Boyd, and M. E. Ridgeway, J. Colloid and Interface Sci. 296, 41 (2006).

[9] W. Wei, Y. Liu, Y. Wei, K. Jiang, L.-M. Peng, and S. Fan, Nano Lett. 7, 64 (2007). 